Apidologie, 1975, 6 (4), 361-380.

\title{
DIE ABHÄNGIGKEIT DER TEMPERATURREGULIERUNG VON DER GENETISCHEN VARIABILITÄT DER HONIGBIENE (APIS MELLIFICA)*
}

\author{
Relations entre la thermorégulation et la variabilité génétique \\ chez l'Abeille (Apis mellifica L.)
}

\author{
Dorothea BRÜCKNER \\ Zoologisches Institut der Universität, 8 München 2, Luisenstr. 14 und \\ Institut für Bienenkunde 637 Oberursel / Ts., Im Rothkopf 5
}

\begin{abstract}
SUMMARY
THE INFLUENCE OF GENETIC VARIABILITY ON THERMOREGULATION

IN HONEYBEES
\end{abstract}

The thermoregulation of inbred and non-inbred colonies of the honeybee (Apis mellifica) was compared. This trait - a measure of physiological homeostasis - was chosen to determine if homeostatic abilities are bound to heterozygosity in haplo-diploid systems. All inbred colonies had been inbred by artificial insemination in the second generation (Inbreeding coefficients $=87,5 \%$ ). Only the reaction to low environmental temperatures was determined in the experiments. The broodnest temperatures of colonies outdoors were measured in early spring and the temperatures reached by groups of 50 workers were measured, which were kept in cages at environmental temperatures of $15{ }^{\circ} \mathrm{C}, 20{ }^{\circ} \mathrm{C}, 22{ }^{\circ} \mathrm{C}$ and $25{ }^{\circ} \mathrm{C}$. A total of 14 inbred and 9 non-inbred colonies was tested.

Normally heterozygous workers were superior in their thermoregulation compared to related and unrelated highly homozygous workers : they reached significantly higher tempecatures in the broodnest and were able to keep the temperatures more stable. In the experiments with the small groups of workers it was found that normally heterozygous workers produced more metabolic heat than highly homozygous workers. It was also found that the survival rate under coldstress was greater in non-inbred than in inbred workers. It is concluded that the poorer thermoregulation of the inbred colonies is due to their high degree of homozygosity which must affect the loci that are involved in thermoregulation. Thus this trait of homeostasis is bound to heterozygosity in the haplo-diploid system of the honeybee.

* Diese Arbeit ist Teil einer Inaugural-Dissertation zur Erlangung der Doktorwürde an der Universität 
The question is discussed whether there is a change in the sollwert of the inbred bees besides the poorer abilities to regulate the temperature, which is due to a smaller production of metabolic heat.

\section{ZUSAMMENFASSUNG}

Bei Inzucht- und Nicht-Inzuchtvölkern der Honigbiene (Apis mellifica) wurde das Temperaturregulierungsvermögen vergleichend untersucht, um festzustellen, ob die genetische Variabilität der Biene einen Ein fluß auf dieses Merkmal besitzt. Alle untersuchten Inzuchtvölker waren durch künstliche Besamung in der 2. Generation ingezüchtet (Inzuchtkoeffizient $=87,5 \%$ ). Es wurden Messungen an intakten Völkern im Freien sowie an Gruppen von je 50 gekäfigten Arbeiterinnen bei $15{ }^{\circ} \mathrm{C}, 20^{\circ} \mathrm{C}, 22{ }^{\circ} \mathrm{C}$ und $25^{\circ} \mathrm{C}$ durchgeführt. Insgesamt wurden 14. Inzucht-und 9 Nicht-Inzuchtvölker getestet.

Es konnte gezeigt werden, daß normal heterozygote Arbeiterinnen verwandten und nichtverwandten Arbeiterinnen mit hohem Homozygotiegrad in ihrer Fähigkeit zur Temperaturregulierung überlegen sind. Die Nicht-Inzuchtbienen erreichen signifikant höhere Temperaturen als die Inzuchtbienen und können diese im Volk stabiler halten. Außerdem konnte gezeigt werden, daß die Überlebenschance der Nicht-Inzuchtbienen im Kältestreß größer ist als die der Inzuchtbienen. Die schlechtere Temperaturregulierung der Inzuchtarbeiterinnen wird darauf zurückgeführt, daß die Reduktion der genetischen Variabilität das innere Gleichgewicht (physiologische Homöostasis) der Tỉere so stört, daß die Mechanismen der Temperaturregulierung nicht mehr wie bei normal heterozygoten Tieren funktionieren. Von den Inzuchtarbeiterinnen wird zum Beispiel unter gleichen Bedingungen weniger Stoffwechselwärme erzeugt als von den Nicht-Inzuchtarbeiterinnen. Es wird diskutiert, ob neben einer schlechten Regulierfähigkeit auch eine Verschiebung des Sollwerts bei den Inzuchtarbeiterinnen vorliegen könnte.

\section{EINLEITUNG}

Die Fähigkeit zur Temperaturregulierung ist unter den sozialen Insekten bei der Honighiene (Apis mellifica) am besten ausgeprägt. Die als Individuen weitgehend von der Umgebungstemperatur abhängigen Tiere besitzen als Volk ein gut ausgebildetes Temperaturregulierungsvermögen, das in seiner Leistung dem homoiothermer Lebewesen nicht nachsteht. So ist bekannt, daß Bienenvölker ganz extremen Temperaturschwankungen in ihrer Umwelt zu widerstehen vermögen und in der Lage sind, die Temperatur des Brutnestes stets bei etwa $35{ }^{\circ} \mathrm{C}$ zu halten. Dieser Fähigkeit liegt ein Regelkreis zugrunde, in dem ein bestimmter Temperatursollwert gegenüber störenden Außenfaktoren aufrechterhalten wird (WILson 1971) : Setzt man ein Volk unter experimentellen Bedingungen der Kälte aus, so wird vermehrt Stoffwechselwärme von den Individuen erzeugt. Diese Wärme wird bei gleichbleibend niedriger Umgebungstemperatur dadurch erhalten, daß das Bienenvolk eine Traube bildet. Die Traube kann sich je nach Temperatur stärker oder schwächer um das Brutnest zusammenziehen; je stärker sie zusammengezogen ist, desto geringer ist die der Kälte ausgesetzte Oberfläche, dabei bildet sich gewissermaßen eine lebende Decke von Bienen im äußeren Bereich der Kugel, durch die kalte Luft am Eindringen ins Zentrum der Kugel gehindert wird (Gates 1914, Нrмmer 1932). Bei Überhitzung setzt erhöhtes Flügelfächeln ein, das der Zirkulation der Luft im Stock dient und den Austausch von Luft durch das 
Flugloch beschleunigt. Als weiteres Mittel wird die Verdunstungskälte ausgenutzt, indem die Bienen Wassertröpfchen in den Stock eintragen und sie, als dünne Schicht ausgebreitet, in den Zellen verdunsten lassen (Lindauer 1954). Einige von der Biene eingesetzte Mechanismen sind somit ähnlich denen homoiothermer Lebewesen. Diese erreichen bei großer Kälte z.B. durch Muskelzittern eine Erhöhung der Abgabe an Stoffwechselwärme und nutzen bei großer Hitze durch Schweißabgabe ebenfalls die Verdunstungskälte aus, um abzukühlen.

Die Untersuchungen der vorliegenden Arbeit behandeln nur die kältebedingten Reaktionen der Bienen. Das Temperaturregulierungsvermögen der Honigbiene beruht einerseits auf Reaktionen der einzelnen Individuen, andererseits auf Reaktionen des Kollektivs aller Tiere. Bei der Temperaturregulierung, die bei niedrigen Umgebungstemperaturen einsetzt, wird z. B. vom Individuum die Stoffwechselwärmeabgabe erhöht, während durch das Zusammenwirken aller Tiere die Traubenbildung zustande kommt.

Die vorliegende Arbeit befaßt sich mit der Frage, ob und inwieweit das Temperaturregulierungsvermögen der Bienen, als Ausdruck der physiologischen Homöostasis, von der Heterozygotie der Arbeiterinnen abhängig ist. Von diploiden Arten ist bekannt, daß die Heterozygotie der Tiere eine große Rolle für die homöostatischen Mechanismen der Tiere spielt (Lerner 1954). Im haplo-diploiden System bringen homöostatische Mechanismen, die an Heterozygotie gebunden sind, jedoch nur dem diploiden Teil der Population einen Vorteil. Es wäre daher denkbar, daß die Homöostasis in haplo-diploiden Systemen nicht an Heterozygotie gebunden ist.

Mit Untersuchungen an Isoenzymen konnte gezeigt werden, daß bei der Honigbiene eine Reduktion der genetischen Variabilität in den Populationen vorliegt. Bei Arbeiterinnen und Drohnen von vier verschiedenen Rassen der Honigbiene wurden drei Enzyme auf Polymorphismen hin untersucht (Malat-Dehydrogenase, Phospho-gluco-mutase und Esterase). Von zahlreichen diploiden Arten sind die untersuchten Enzyme als polymorph bekannt, bei der Biene konnten jedoch keine Polymorphismen nachgewiesen werden (BR̈̈́cKNER 1973 a). Diese Reduktion der genetischen Variabilität kann darauf zurückgeführt werden, daß im hemizygoten Chromosomensatz der Drohnen alle Gene der Selektion ausgesetzt sind und sich keine heterotischen Mechanismen ausbilden können. Die Fixierung optimaler Enzyme wird zudem durch die relativ konstanten Umweltbedingungen im Stock begünstigt.

Untersuchungen zur Homöostasis der Biene haben andererseits gezeigt, daß die Entwicklungshomöostasis der Individuen auch im haplo-diploiden System an die Heterozygotie der Tiere gebunden ist (Brückner 1975). Als Maß für die Entwicklungshomöostasis diente die Flügelsymmetrie der Individuen. Störungen während der Ontogenese führen zu erhöhter morphologischer 
Variabilität des adulten Tieres; durch homöostatische Mechanismen wird diese Variabilität reduziert. Die Konstanz eines Merkmals kann als Maß für Homöostasis genommen werden, sofern die Konstanz für das Merkmal von adaptivem Wert ist (Lewontin 1956). Symmetrische Flügel sind von größter Wichtigkeit für Arbeiterinnen und Drohnen; die Arbeiterinnen müssen große Flugleistungen erbringen, um Futter für die Kolonie zu suchen, und die Drohnen, um die Drohnensammelplätze zu erreichen. Die Differenz zwischen rechten und linken Flügeln wurde aus Messungen an drei morphogenetischen Merkmalen für Arbeiterinnen und Drohnen berechnet. Es stellte sich heraus, daß Arbeiterinnen, deren genetische Variabilität durch Inzucht stark verringert war, in ihrer Flügelsymmetrie variabler waren als verwandte nicht-ingezüchtete Arbeiterinnen; die Drohnen waren stets variabler als die Arbeiterinnen (BrücKNER 1975). Mit demselben Versuchsmaterial, sowie anderen Inzucht-und Nicht-Inzuchtvölkern, wurde in der vorliegenden Arbeit getestet, inwieweit sich auch in der physiologischen Homöostasis Unterschiede zwischen den beiden Gruppen nachweisen lassen.

\section{MATERIAL}

Als Versuchsmaterial dienten ingezüchtete und nicht-ingezüchtete Völker von Apis mellifica carnica, von denen einige eng verwandt waren, während andere unterschiedlichen Linien entstammten. Insgesamt wurde mit 14 Inzucht- und 9 Nicht-Inzuchtvölkern gearbeitet. Die Art der Inzucht für die verwandten Völker ist in Abb. 1 wiedergegeben (die Königinnen und ihre Völker wurden jeweils gleich bezeichnet). Alle in den Versuchen getesteten Inzuchtvölker waren mit Hilfe der künstlichen Besamung in der zweiten Generation ingezüchtet. Nach dieser Art der Inzucht rechnet man bei den Völkern mit einem Inzuchtkoeffizienten $F$ von $87,5 \%$ (Drescher 1969); der Inzuchtkoeffizient entspricht dem Homozygotiegrad der Tiere. Da der Locus der Sexallele stets heterozygot sein muß, um Arbeiterinnen entstehen zu lassen, können bei der Honigbiene keine völlig homozygoten Linien hergestellt werden (Mackensen 1951, Woyke 1965). Die Nicht-Inzuchttiere stammten von Völkern, deren Königinnen sich frei gepaart hatten (Inzuchtkoeffizient $=0 \%$ ). Alle Versuche wurden im Frühjahr 1974 von März bis Juni im Institut für Bienenkunde in Oberursel bei Frankfurt durchgeführt.

\section{METHODE}

Es wurden Temperaturmessungen in intakten Völkern sowie an Gruppen von je 50 gekäfigten Arbeiterinnen durchgeführt. Für die Messungen in den Völkern wurde ein elektronisches Messgerät (Polycomb) benutzt, an das 6 Thermoelemente angeschlossen waren. Diese konnten zu den im Freien stehenden Völkern geführt und dort so auf den Wabenrähmchen befestigt werden, daß die feinen Spitzen der Elemente in Zellen mit verdeckelter Brut eingesteckt werden konnten.

In 4 aufeinanderfolgenden Versuchsserien wurde jeweils ein Nicht-Inzuchtvolk mit einem oder zwei Inzuchtvölkern verglichen. Vor Versuchsbeginn wurden die Völker, die alle in Magazin-Beuten (einwandige Magazine im Zandermaß) untergebracht waren, auf etwa gleiche Stärke gebracht, d.h. daß die gleiche Anzahl an Wabenrähmchen etwa gleich dicht besetzt war. Die Magazine wurden am Versuchsort direkt nebeneinander aufgestellt. Die Bienen konnten sich dann mehrere Stunden lang ein fliegen, ehe die Thermoelemente in den Völkern befestigt wurden. Es wurde darauf geachtet, daß die Thermoelemente an sich entsprechenden Stellen in den Völkern angebracht wurden, und zwar jeweils im Zentrum des Brutnestes. Zur vergleichenden Messung der Umgebungstemperatur wurde bei jeder Versuchsserie ein Thermo- 


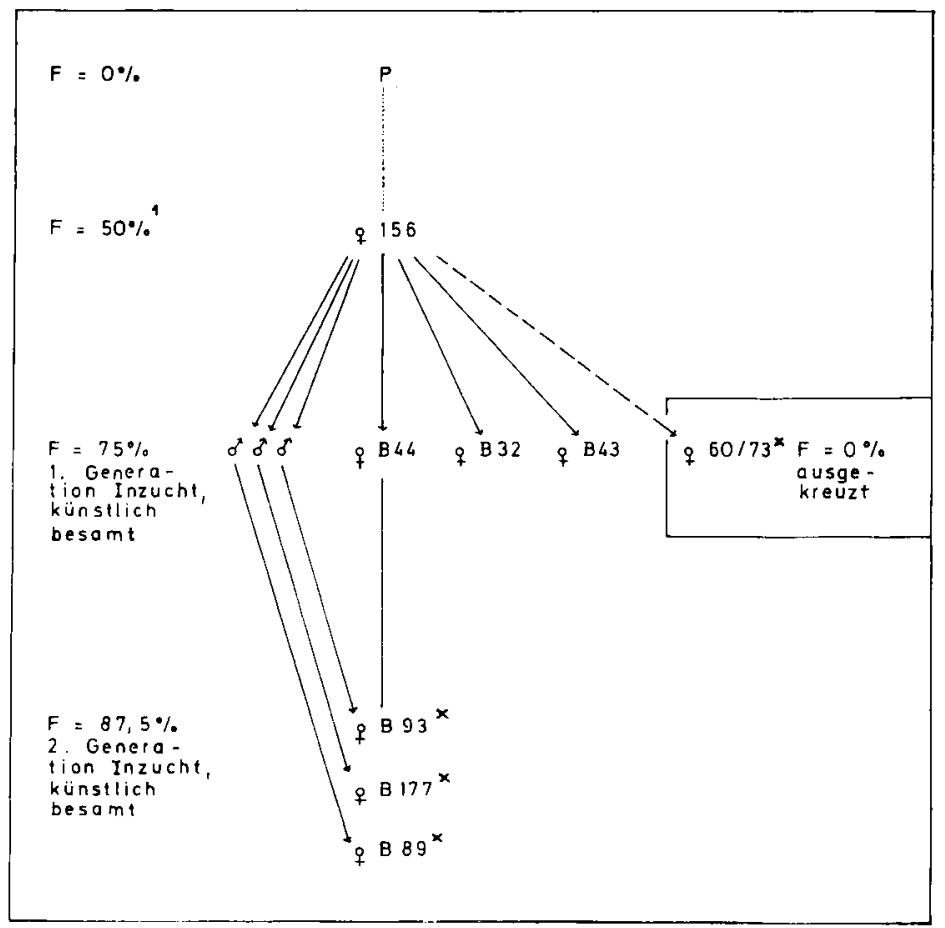

Авв. 1. - Art der Inzucht und Inzuchtkoeffizient $F$ bei den verwandten Völkern

$\times$ Völker in den Versuchen

1 Zwischen der Generation $P$ und 156 liegen mehrere Generationen, bei denen die Königinnen auf einer Belegstelle durch Kreuzung mit eng verwandten Drohnen ingezïchtet wurden, um den Inzuchtkoeffizienten $\mathbf{F}=50 \%$ zu erreichen.

Fig. 1. - Type de consanguinité et coefficient de consanguinité F chez les colonies apparentêes.

$\times$ colonies en expérience

1 entre les générations $\mathrm{P}$ et 156 il y a plusieurs générations dont les reines ont été croisées en station de fécondation avec des mâles de parenté suffisamment proche pour que le coefficient de consanguinité atteigne $50 \%$.

element unmittelbar außerhalb der nebeneinanderstehenden Magazine befestigt, und zwar so, daß es sich stets im Schatten befand. Auf dem an das Meßgerät angeschlossenen Schreiber wurde ein Papiervorschub von $2 \mathrm{~cm}$ in der Stunde und eine Punktfolge von 12 sec eingestellt. Der statistischen Auswertung der Temperaturmessungen in den Völkern liegen Meßwerte zugrunde, die in zweistündigem Abstand über eine Zeitdauer von jeweils 24 Stunden abgelesen wurden. Es wurde allerdings erst 4 Stunden nach dem Beginn der Messungen bzw. dem Anbringen der Thermoelemente mit dem Ablesen der Daten begonnen, um etwaige Störreaktionen auszuschließen, die durch das Anbringen der Thermoelemente hätten verursacht sein können. Insgesamt wurde die Temperatur in 7 Inzucht- und 3 Nicht-Inzuchtvölkern gemessen.

Für die Versuche mit den gekäfigten Bienen wurden Waben mit verdeckelter Brut aus den Völkern entnommen, und die Bienen im Brutschrank bei $35^{\circ} \mathrm{C}$ zum Schlüpfen gebracht. Die jungen Arbeiterinnen wurden innerhalb der ersten Stunden nach dem Schlüpfen von der Wabe abgesammelt und zu je 50 von einem Volk in ein Plexiglaskästchen gesetzt. Dieses hatte die Innenmaße $9 \times 6 \times 5 \mathrm{~cm}$ und war nach Free (1958) verändert gebaut (Abb. 2). Durch die Vorderfront konnten 2 Pipetten eingeführt werden, von denen die eine Honig, die andere 


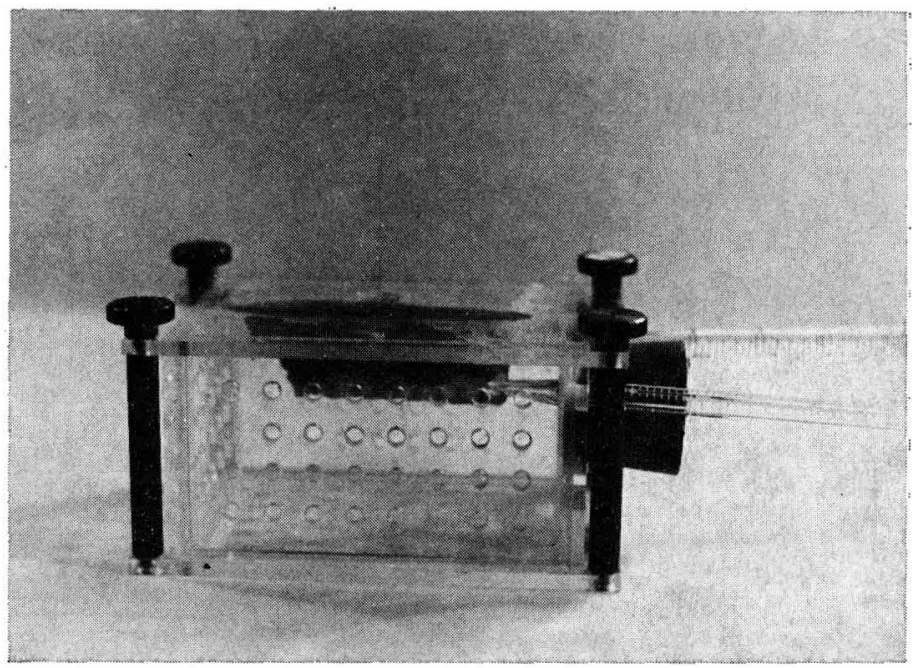

Aвв. 2. - Kästchen nach Free

FIG. 2. - Cagette type Free

Wasser enthielt; an den 3 anderen Seiten befanden sich zahlreiche Luftlöcher. Um den Bienen einen Halt zu geben, war ein $5 \times 1 \mathrm{~cm}$ langes Stück Wachsmittelwand mit flüssigem Wachs an die Decke des Kästchens geklebt worden.

Die Bienen wurden 9 Tage lang in diesen Kästchen im Brutschrank bei $28{ }^{\circ} \mathrm{C}$ im Dauerdunkel gehalten. Diese Zeitspanne zwischen Schlüpfen der Bienen und Versuchsbeginn wurde gewählt, um die Bienen in ein Alter kommen zu lassen, in dem sie, wären sie im Volk geblieben, als Ammenbienen für das Warmhalten der Brut tätig sein würden. Während dieser Zeit sowie während der Versuche stand den Bienen Honig und Wasser in beliebiger Menge zur Verfügung. Am ersten Tag nach dem Schlüpfen erhielten die Bienen in jedem Kästchen eine Gabe von $4 \mathrm{~g}$ frischem Pollen. Am 10. Tag wurden die Bienen in Klimakammern der Temperaturstufen $15^{\circ}$, $20^{\circ}, 22^{\circ}$ und $25^{\circ} \mathrm{C}$ gebracht, in denen gelbes Dauerlicht brannte. Die Messungen begannen nach einer Eingewöhnungszeit von einer Stunde und wurden mit einem transportablen Meßgerät (Braun Typ AAHT1) durchgeführt, das einen Stechfühler besaß $(\varnothing 18 \mathrm{~mm})$, der durch die seitlichen Luftlöcher in die Kästchen eingeführt werden konnte; mit diesem konnte jeweils die wärmste Stelle im Kästchen gemessen werden, ohne unter den Bienen größere Störungen zu verursachen. Die Versuche dauerten 3 bis 4 . Tage, an denen die Temperaturen stündlich zwischen 8 und $18 \mathrm{Uhr}$ in allen Kästchen gemessen wurde. Die Umgebungstemperatur wurde von einem Thermometer abgelesen, das unmittelbar neben den Kästchen befestigt war.

Bei den Temperaturstufen $15^{\circ}, 20^{\circ}$ und $25{ }^{\circ} \mathrm{C}$ wurden Arbeiterinnen eines Inzuchtvolkes (B93) und eines verwandten Nicht-Inzuchtvolkes $(60 / 73)$ vergleichend gemessen, und zwar je 2 Kästchen pro Volk nebeneinander. Bei $22^{\circ} \mathrm{C}$ wurden verschiedene Inzucht-und NichtInzuchtvölker, jeweils nur ein Kästchen pro Volk, auf ihre Temperaturregulierung hin geprüft.

Nach diesen Messungen zur Temperaturregulierung wurden die Bienen in den Kästchen für 2 Tage in einem warmen Brutschrank $\left(28{ }^{\circ} \mathrm{C}\right)$ gehalten und dann einem Kältestress unterworfen, der ihre Uberlebensfähigkeit zeigen sollte. Die Bienen, die während der Versuche den Temperaturstufen $15^{\circ}, 20^{\circ}$ und $25{ }^{\circ} \mathrm{C}$ ausgesetzt waren, wurden 48 Stunden bei $13{ }^{\circ} \mathrm{C}$ gehalten, während die der Temperaturstufe $22{ }^{\circ} \mathrm{C}$ für 12 Stunden bei $11{ }^{\circ} \mathrm{C}$ gehalten wurden. Danach wurde die Zahl der überlebenden Bienen festgestellt. Insgesamt wurden in den Kästchenversuchen Bienen aus 9 Inzucht-und 5 Nicht-Inzuchtvölkern getestet.

Bei der statistischen Auswertung wurden alle Werte, die unter $5 \%$ Irrtumswahrscheinlichkeit liegen, als signifikant bezeichnet. 


\section{ERGEBNISSE}

\section{Temperaturmessungen in den Völkern}

Bei den Messungen in den Völkern stellte sich heraus, daß die NichtInzuchtbienen trotz unterschiedlicher Umgebungstemperaturen die Temperatur des Brutnestes stets zwischen $34{ }^{\circ} \mathrm{C}$ und $35{ }^{\circ} \mathrm{C}$ hielten. Da die Brutnesttemperatur relativ konstant gehalten wird, nehmen die Differenzen zwischen Brutnesttemperatur und Umgebungstemperatur mit steigender Umgebungstemperatur ab. Die größte Heizleistung der Völker in den Versuchen wurde daher bei der niedrigsten Umgebungstemperatur erbracht. Bei einer Umgebungstemperatur von im Mittel 2,2 ${ }^{\circ} \mathrm{C}$ hielt ein Nicht-Inzuchtvolk eine Temperaturdifferenz von im Mittel $32,17^{\circ} \mathrm{C}$ aufrecht (Abb. 3).

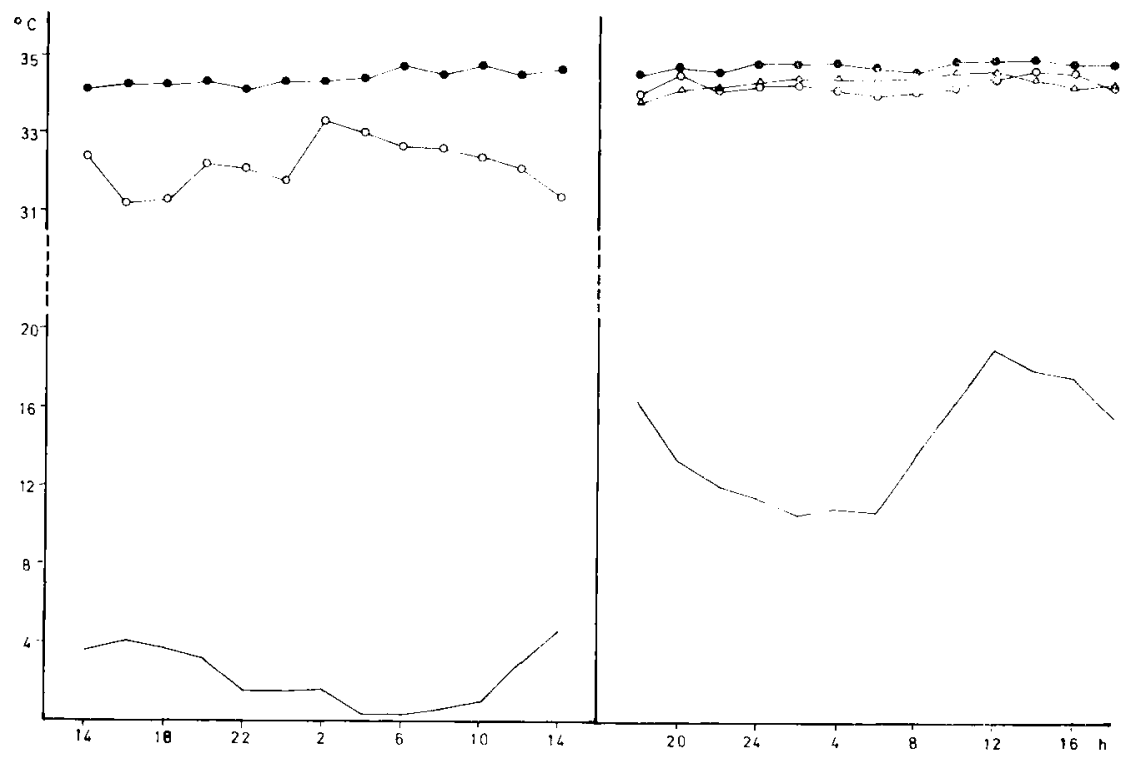

Авв. 3. - In den Brutnestern erreichte Temperaturen verschiedener Inzucht-und Nicht-Inzuchtvölker bei niedriger und höherer Umgebungstemperatur (Messungen vom 15/16.3 und 18/19.4.1974)

(Inzuchtvölker, $\mathrm{N}=3$; Nicht-Inzuchtvölker $\mathrm{N}=2$ )

Inzucht $\mathrm{O}_{-}-\mathrm{Q}_{\mathrm{J}}$ Nicht-Inzucht $\bullet \ldots \ldots$ Umgebungs-

Fig. 3. - Températures atteintes dans le nid à couvain de diverses colonies consanguines et non consanguines dans 2 cas de température ambiante, l'une basse, l'autre élevée. (Mesures du 15-16-3 et du 18-19.3.1974). Consanguinité - Température

Beim Vergleich der Brutnesttemperatur von Inzucht- und Nicht-Inzuchtvölkern stellten sich Unterschiede in der Leistung heraus. In Abb. 3 sind als 
Beispiel die Temperaturkurven der im Brutnest erreichten Temperaturen verschiedener Inzucht- und Nicht-Inzuchtvölker am Tage der niedrigsten und am Tage der höchsten Umgebungstemperatur der Versuchsserie wiedergegeben. Bei beiden Messungen erreichen die Inzuchtvölker niedrigere Temperaturen als die Nicht- Inzuchtvölker. Während von der niedrigen zur höheren Umgebungstemperatur die im Mittel erreichte Temperatur bei den Inzuchtvölkern um etwa $2{ }^{\circ} \mathrm{C}$ zunimmt, ändert sich die erreichte Temperatur der NichtInzuchtvölker nur um Bruchteile von Graden. Die Schwankungen der erreichten Temperatur sind zudem bei den Inzuchtvölkern bei niedriger und höherer Umgebungstemperatur größer als bei den Nicht-Inzuchtvölkern. Vergleicht man die erreichten Temperaturen aller Inzucht- und Nicht-Inzuchtvölker dieser Versuchsserie, so stellt sich heraus, daß die im Mittel erreichten Temperaturen der Nicht-Inzuchtvölker in 6 von 7 Fällen über denen der Inzuchtvölker liegen, davon in 5 Fällen signifikant, während nur in einem Fall die erreichte Temperatur der Inzucht signifikant über der der Nicht-Inzucht liegt. Vergleicht man die Varianzen der erreichten Temperaturen aller Völker in den Versuchen, so stellt sich heraus, daß die der Inzucht in 5 von 7 Fällen größer sind als die der Nicht-Inzucht, davon in 3 Fällen signifikant größer. In 2 Fällen haben die Inzuchtvölker geringere Varianzen als die Nicht-Inzuchtvölker, jedoch sind die Werte nicht signifikant verschieden. Stellt man die gemeinsame Varianz in der erreichten Temperatur der Inzuchtvölker der gemeinsamen Varianz in der erreichten Temperatur der Nicht-Inzuchtvölker gegenüber, so zeigt die Brutnesttemperatur der Inzuchtvölker signifikant $(\mathrm{p}<0,01)$ mehr Fluktuationen als die der Nicht-Inzuchtvölker. Die NichtInzuchtarbeiterinnen sind also in der Lage, die Brutnesttemperatur höher und stabiler zu halten als die Inzuchtarbeiterinnen.

Die im Brutnest erreichten Temperaturen der Inzuchtvölker sind mit den Werten der Umgebungstemperatur positiv korreliert $(r=0,606, N=85$, $\left.\mathbf{B}=\mathbf{r}^{2}=0,367, \mathbf{p}<0,01\right)$. Liegen die Umgebungstemperaturen höher, so liegen auch die in den Völkern erreichten Temperaturen höher (Abb. 4). Bei diesem Vergleich wird die Gruppe der Inzuchtvölker $(F=87,5 \%)$ der Gruppe der Nicht-Inzuchtvölker $(F=0 \%)$ gegenübergestellt. Die im Brutnest der Nicht-Inzuchtvölker erreichten Temperaturen sind nicht mit den Werten der Umgebungstemperatur korreliert. Die bei den Inzuchtvölkern in dieser Korrelation gefundene Variabilität der erreichten Temperaturen läßt sich mit $36,7 \%$ auf die Variabilität der Umgebungstemperatur zurückführen (Bestimmtheitsmaß $\mathrm{B}=0,367$ ).

Wenn man die Annahme ausschließt, daß es sich bei dem Inzuchtvolk, das bei der niedrigsten Umgebungstemperatur gemessen wurde, um ein irgendwie krankes Volk handelte, so muß man folgendes aus den Messungen in den Völkern schließen : Die starke Abhängigkeit der Inzuchtvölker von der Umgebungstemperatur bedingt, daß diese Völker im zeitigen Frühjahr bei niedrigen 


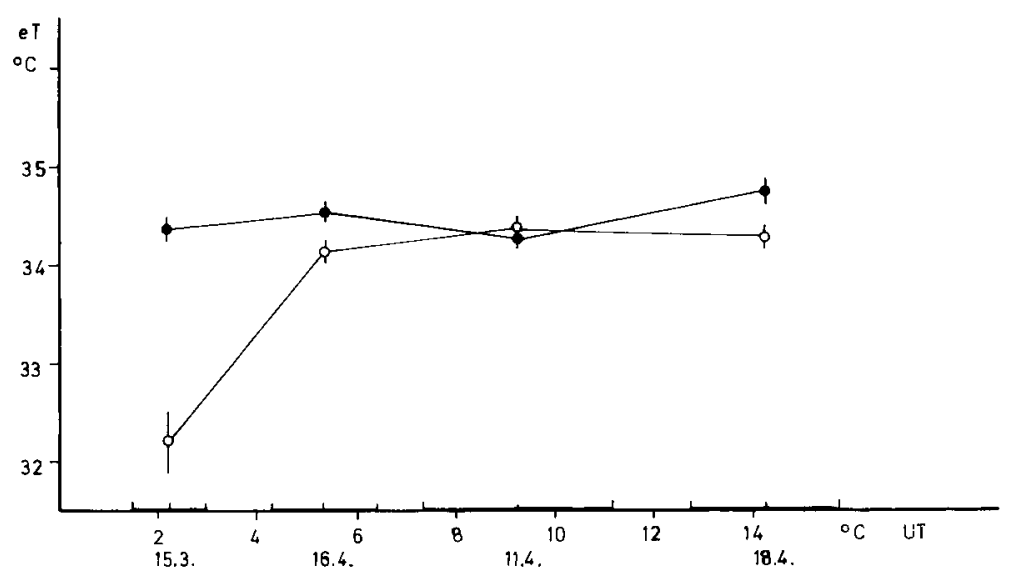

Aвв. 4. - Mittelwerte mit Standardabweichungen der erreichten Temperaturen (eT) in den Völkern bei steigenden Mittelwerten der Umgebungstemperatur (UT)

(Inzuchtvölker, $\mathbf{N}=7$; Nicht-Inzuchtvölker, $\mathbf{N}=3$ )

Inzucht $\bigcirc-\cdots, 0$ Nicht-Inzucht $\bullet-\cdots$

FIG. 4. - Moyennes avec les écarts-types des températures atteintes (eT) dans les colonies lorsque la moyenne de la température ambiante (UT) croil.

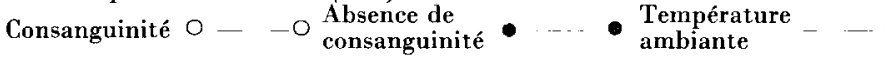

Umgebungstemperaturen die erforderliche Brutnesttemperatur von $34^{\circ}$ bis $35{ }^{\circ} \mathrm{C}$ nicht erreichen können und deshalb dann keine Brut aufziehen können, während die Nicht-Inzuchtvölker dazu in der Lage sind.

\section{Temperaturmessungen in den Kästchenversuchen}

In den Kästchenversuchen wurden die Temperaturen kleiner Gruppen von Bienen außerhalb des Sozialverbandes gemessen, um die Leistung der einzelnen Individuen besser zu erfassen. Wenn die Gruppen sehr klein sind und keine Brut vorhanden ist, haben die Bienen kaum die Tendenz, eine Traube zu bilden (Free 1959). Die Traubenbildung stellt im Volk die soziale Komponente der Reaktionen auf niedrige Umgebungstemperaturen dar. Da Traubenbildung bei Gruppen von 50 Arbeiterinnen in den Kästchenversuchen praktisch nicht vorkommt, wird in diesen Versuchen die Leistung der Individuen bei der Erzeugung von Stoffwechselwärme gemessen.

Im Verhalten der Bienen wurde beobachtet, da $\beta$ die Inzuchtarbeiterinnen in den Kästchenversuchen oft weniger Aktivität zeigten als die Nicht-Inzuchtarbeiterinnen.

Einige Bienengruppen hatten in der Zeit zwischen Schlüpfen und Versuchsbeginn (10 Tage) damit begonnen, das vorgegebene Stück Mittelwand an der Decke der Kästchen auszubauen (Abb. 5) : 9 von 10 Gruppen von Nicht-Inzucht- 


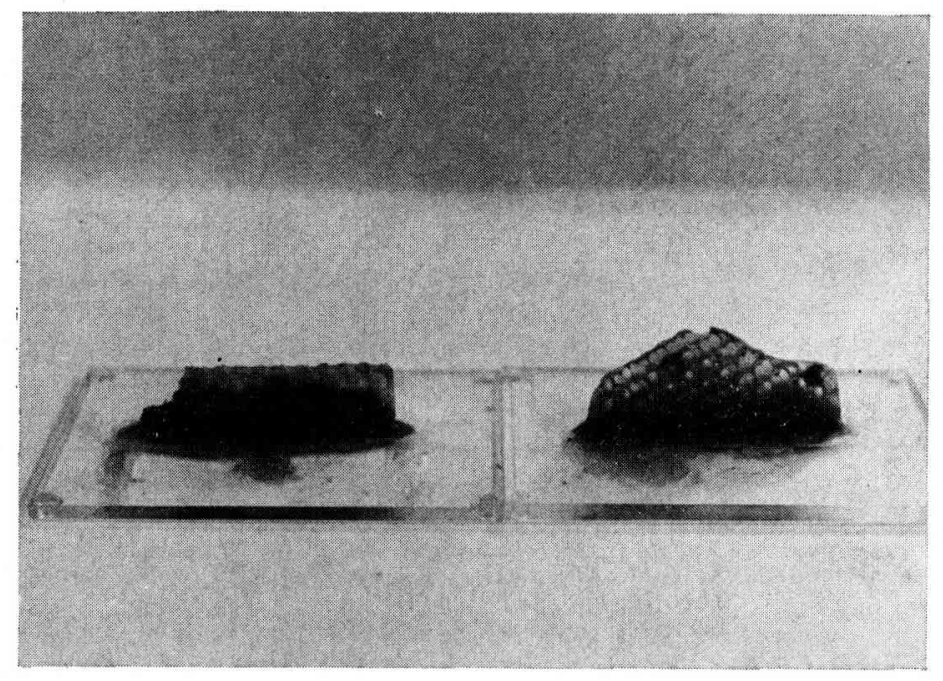

Aвв. 5. - Vorgegebenes Stück der Mittelwand (links) und Anfänge von Wabenbau (reehts). Fig. 5. - Morceau de cire gaufrée fourni aux abeilles (à gauche) et dêbuts de construction (à droite).

arbeiterinnen hatten mit dem Wabenbau begonnen, während nur 2 von 14 Inzuchtgruppen Anfänge von Wabenbau zeigten. Die Tatsache, daß die Gruppen der Inzuchtarbeiterinnen kaum Wabenbau zeigten, könnte entweder daran liegen, daß sich bei den Inzuchtarbeiterinnen die Wachsdrüsen langsamer entwickeln als bei den Nicht-Inzuchtarbeiterinnen, oder aber daran, daß sie weniger gut als Kollektiv geordnet zu arbeiten in der Lage sind.

Bei den Temperaturmessungen in den Kästchen erreichten die Arbeiterinnen in den verschiedenen Temperaturstufen nie Temperaturen von $34^{0}$ bis $35^{\circ} \mathrm{C}$, wie sie im Brutnest der Völker erreicht wurden. Jedoch zeigte sich auch in den Kästchenversuchen die außerordentlich große Heizleistung der Bienen. So waren 50 Arbeiterinnen in der Lage, eine Umgebungstemperatur von $15{ }^{\circ} \mathrm{C}$ über 12 Stunden um etwa $10^{\circ} \mathrm{C}$ aufzuheizen (Abb. 6). In Abb. 6, 7 und 8 sind als Beispiel die erreichten Temperaturen von Arbeiterinnen eines Inzucht-und eines Nicht-Inzuchtvolkes bei Umgebungstemperaturen von $15^{\circ}, 20^{\circ}$ und $25^{\circ} \mathrm{C}$ wiedergegeben. Im Vergleich zwischen Inzucht- und Nicht-Inzuchtarbeiterinnen stellt sich heraus, daß die Nicht-Inzuchtarbeiterinnen höhere Temperaturen erreichen als die Inzuchtarbeiterinnen. Diese Unterschiede sind bei $20^{\circ}$ und $25^{\circ} \mathrm{C}$ signifikant, während sie bei $15^{\circ} \mathrm{C}$ nicht signifikant sind.

Vergleicht man die im Mittel erreichten Temperaturen von Inzucht- 


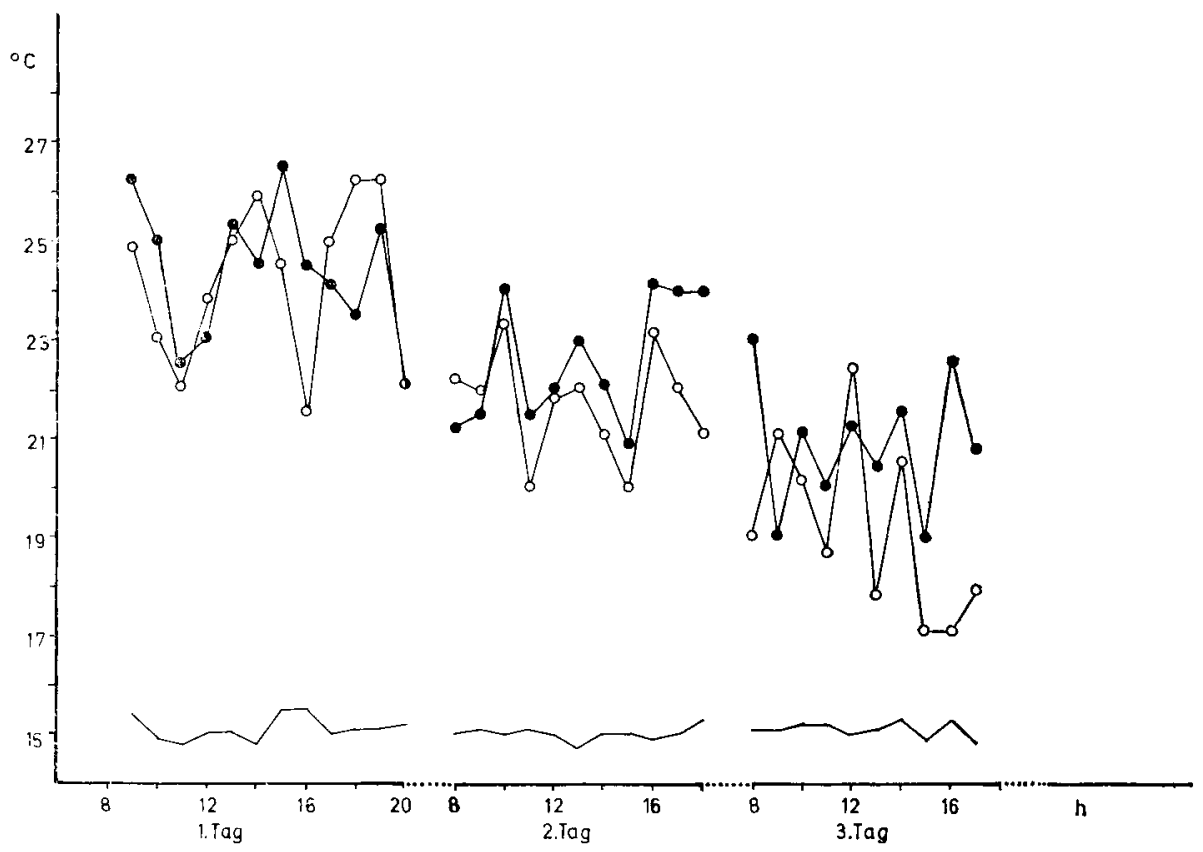

Aв8. 6. - Kästchenversuche : Von Inzucht- und Nicht-Inzuchtarbeiterinnen erreichte Temperaturen bei einer Umgebungstemperatur von $15{ }^{\circ} \mathrm{C}$ Inzucht $\mathrm{O}-\mathrm{O}$ Nicht-Inzucht $-\ldots$ Ungebungs-

Fig. 6. - Essai en cagette : températures atteintes par des abeilles consanguines et non consanguines avec une température ambiante de $15^{\circ} \mathrm{C}$.
Consanguinité $O$.
Absence de
- Température

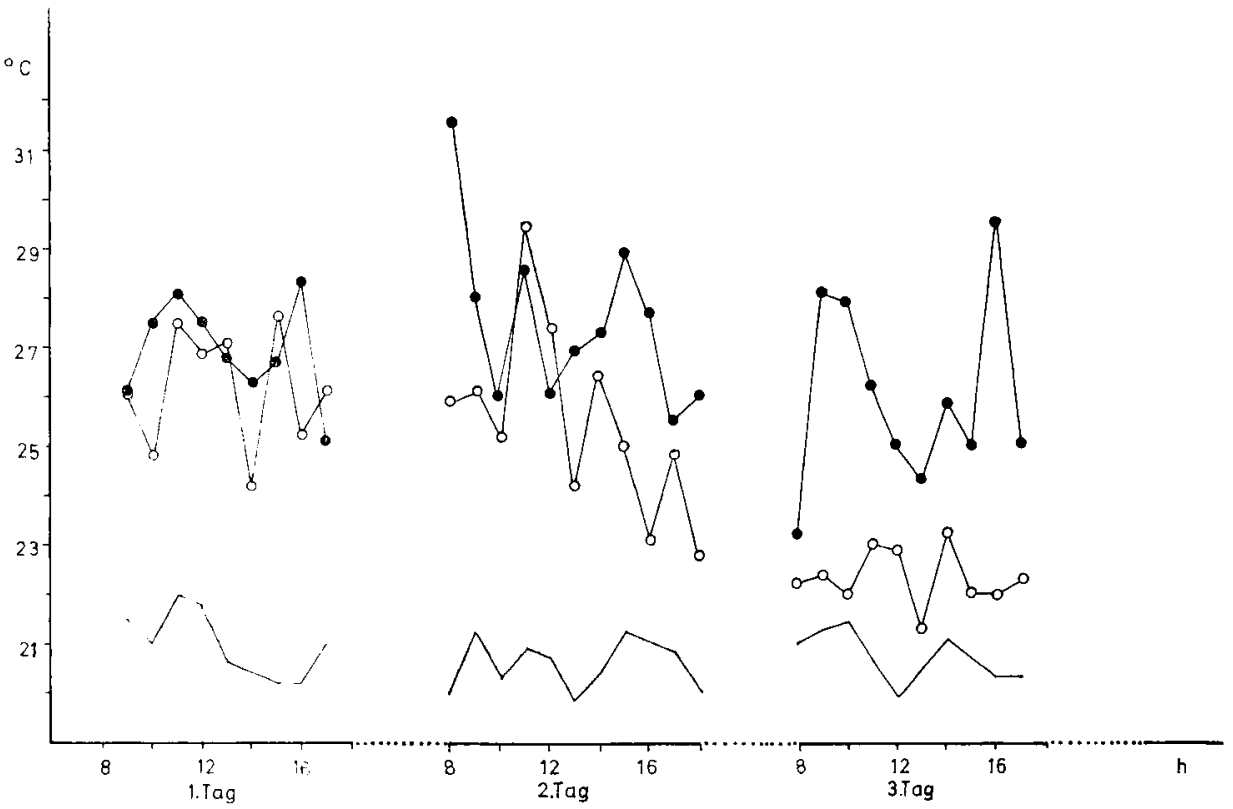

Aвв. 7: - Kästchenversuche : Von Inzucht- und Nicht-Inzuchtarbeiterinnen erreichte Temperaturen bei einer Umgebungstemperatur von $20 \mathrm{C}^{\circ}$
Inzucht $O$
O Nicht-Inzucht
Umgebungs-
temperatur

FIG. 7. - Essai en cagette : températures atteintes par des abeilles consanguines et non consanguines avec une température ambiante de $20{ }^{\circ} \mathrm{C}$.

Consanguinité

Absence de consanguinité

Température
ambiante 


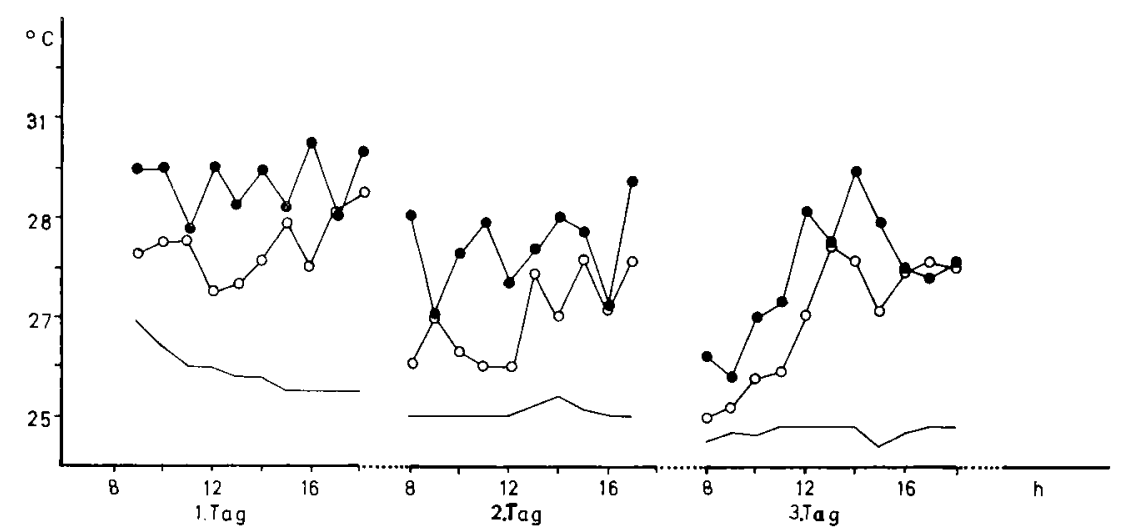

Aвв. 8. - Kästchenversuche : Von Inzucht- und Nicht-Inzuchtarbeiterinnen erreichte Temperaturen bei einer Umgebungstemperatur von $25^{\circ} \mathrm{C}$

Inzucht $O \ldots \ldots$ Nicht-Inzucht $\bullet \ldots \ldots \ldots$ Umgebungs-

FIG. 8. - Essai en cagette : températures atteintes par des abeilles consanguines et non consanguines avec une température ambiante de $25{ }^{\circ} \mathrm{C}$.

Consanguinité $\bigcirc \quad-\cdots \quad \bigcirc \begin{aligned} & \text { Absence de } \\ & \text { consanguinité }\end{aligned}-\longrightarrow-\begin{aligned} & \text { Température } \\ & \text { ambiante }\end{aligned}$

arbeiterinnen und Nicht-Inzuchtarbeiterinnen der gesamten Versuchsserie, so liegen die der Nicht-Inzuchtarbeiterinnen in 10 von 14 Fällen über denen der Inzuchtarbeiterinnen; der Unterschied ist in 7 Fällen signifikant. In 2 Fällen sind die erreichten Temperaturen von Inzucht- und Nicht-Inzuchtarbeiterinnen gleich; in 2 Fällen erreichen die Inzuchtarbeiterinnen höhere Temperaturen, jedoch sind die Unterschiede nicht signifikant. Berechnet man den Unterschied in der erreichten Temperatur von Inzucht- und Nicht-Inzuchtarbeiterinnen für alle Kästchenversuche, so liegen die Werte der NichtInzucht im Mittel um $+0,72^{\circ} \mathrm{C}$ über denen der Inzucht $(p<0,05)$.

Die auftretenden Schwankungen der erreichten Temperaturen sind in den Kästchenversuchen im allgemeinen größer als bei den Messungen im Brutnest (vergl. Abb. 6, 7 und 8 mit Abb. 3); zwischen Inzucht- und Nicht-Inzuchtarbeiterinnen besteht kein signifikanter Unterschied in der Größe dieser Schwankungen. Die Tatsache, daß die erreichten Temperaturen in den Kästchen große Schwankungen aufweisen, ist damit zu erklären, daß die Traubenbildung in den Kästchen nicht zur Wirkung kommen kann. Diese ist im Volk der Hauptfaktor für das Stabilhalten der erreichten Temperaturen.

Die in den Kästchenversuchen von den Arbeiterinnen erreichten Temperaturen sind negativ mit der Zeit korreliert, das heißt, daß die Heizleistung der Bienen in allen Temperaturstufen mit der Zeit abnimmt. Vergleicht man die Werte der Messungen bei $15^{\circ} \mathrm{C}, 20^{\circ} \mathrm{C}$ und $25^{\circ} \mathrm{C}$, bei denen jeweils Arbei- 
terinnen des gleichen Inzucht- und Nicht-Inzuchtvolkes vergleichend gemessen wurden, so stellt sich heraus, daß bei Arbeiterinnen beider Völker die Abnahme der erreichten Temperatur mit der Zeit mit zunehmender Umgebungstemperatur geringer wird. In Abb. 9 wurden die Regressionskoeffizienten (b), die

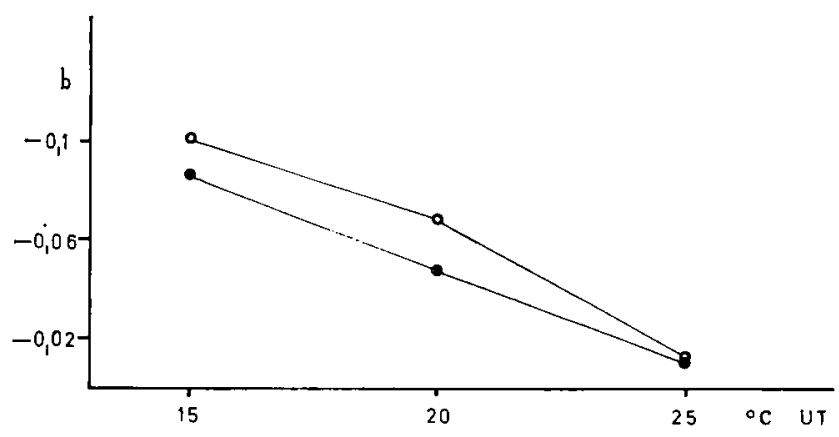

Авв. 9. - Kästchenversuche : Abnehmen der Regressionskoeffizienten b (die die Steilheit des Abfalls der erreichten Temperatur in den Kästchen mit der Zeit angeben) bei Inzucht- und Nicht-Inzuchtarbeiterinnen mit steigender Umgebungstemperatur (UT)

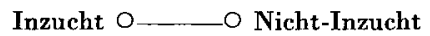

FIG. 9. - Essai en cagette : décroissance des coefficients de régression b (qui indiquent la rapidité de la chute de la température dans les cagettes en fonction du temps) chez des ouvrières consanguines et non consanguines quand la température ambiante croit.

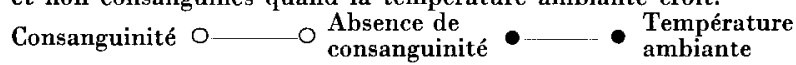

die Steilheit des Abfalls der erreichten Temperaturen in den Kästchen mit der Zeit angeben, gegen die Umgebungstemperatur aufgetragen. Es zeigte sich, daß die Abnahme der erreichten Temperatur mit der Zeit bei den Inzuchtbienen in allen Temperaturstufen größer war als bei den Nicht-Inzuchtbienen. Der Trend zur Abnahme der erreichten Temperaturen wird zweifellos durch den Streß hervorgerufen, den die gewählten Temperaturstufen für die kleinen Gruppen von Bienen bedeuten; der Streß ist bei $15^{\circ} \mathrm{C}$ natürlich am stärksten, daher ist der Abfall bei dieser Temperatur am steilsten.

Bei den Messungen in den 3 verschiedenen Temperaturstufen, $15{ }^{\circ} \mathrm{C}$, $20{ }^{\circ} \mathrm{C}$ und $25{ }^{\circ} \mathrm{C}$, in denen eng verwandte Inzucht und Nicht-Inzuchtarbeiterinnen verglichen wurden, zeigte sich außerdem, daß die Mittelwerte der erreichten Temperaturen bei beiden Gruppen mit steigender Umgebungstemperatur zunehmen (Abb. 10). Dabei besteht bei $15{ }^{\circ} \mathrm{C}$ kein signifikanter Unterschied zwischen Inzucht- und Nicht-Inzuchtbienen, während bei $20^{\circ} \mathrm{C}$ und $25{ }^{\circ} \mathrm{C}$ die Nicht-Inzucht signifikant höhere Werte erreicht. Vergleicht man aber die Differenzen zwischen Umgebungstemperatur und erreichter Temperatur, so nehmen diese mit steigender Umgebungstemperatur bei beiden Gruppen signifikant $(p<0,01)$ ab. In Abb. 10 entspricht der senkrechte Abstand 


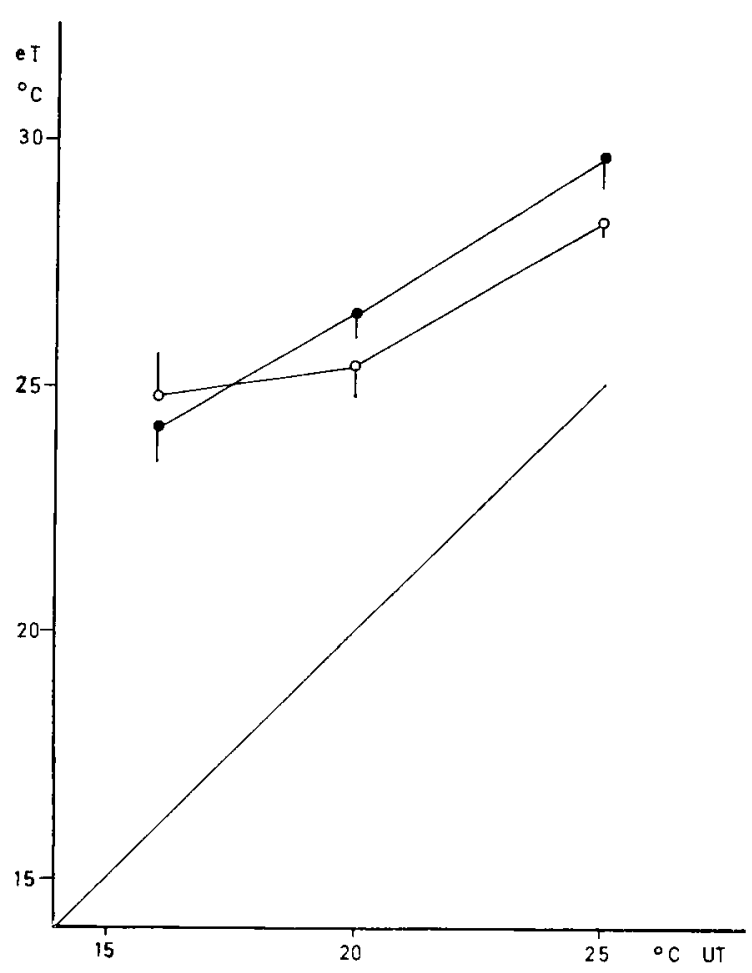

AвB. 10. - Kästchenversuche : Mittelwerte und Standardabweichungen der von Inzucht- und NichtInzuchtarbeiterinnen erreichten Temperaturen (eT) bei steigender Ungebungstemperatur (UT) Inzucht $\mathrm{O}$ - $\mathrm{O}$ Nicht-Inzucht

FIG. 10. - Essai en cagette : Moyennes et écarts-types des températures atteintes par des ouvrières consanguines et non consanguines quand la température ambiante croit.

Consanguinité o Absence de consanguinité Température
ambiante

zwischen den Kurven und der Diagonalen diesen Differenzen. Diese Ergebnisse entsprechen den bei den Messungen im Brutnest der Völker gefundenen Ergebnissen, allerdings sind die im Brutnest der Nicht-Inzuchtvölker erreichten Temperaturen nicht von der Umgebungstemperatur abhängig; als Volk besitzen die Nicht-Inzuchtarbeiterinnen genügend Kapazität, um trotz starker Schwankungen der Umgebungstemperatur die Brutnesttemperatur relativ konstant zu halten.

\section{Versuche zum Kältestress}

Nach den Versuchen zur Heizleisting von kleinen Gruppen von Arbeiterinnen in den Kästchen wurde die Überlebensfähigkeit der Tiere im Kälte- 
Tав. 1. - Vergleich der Überlebensfähigkeit von Inzucht- und Nicht-Inzuchtarbeiterinnen im Kältestress.

TABL. 1. - Comparaison de la capacité de survie des ouvrières consanguines et des ouvrières non consanguines en état de stress thermique (basse température).

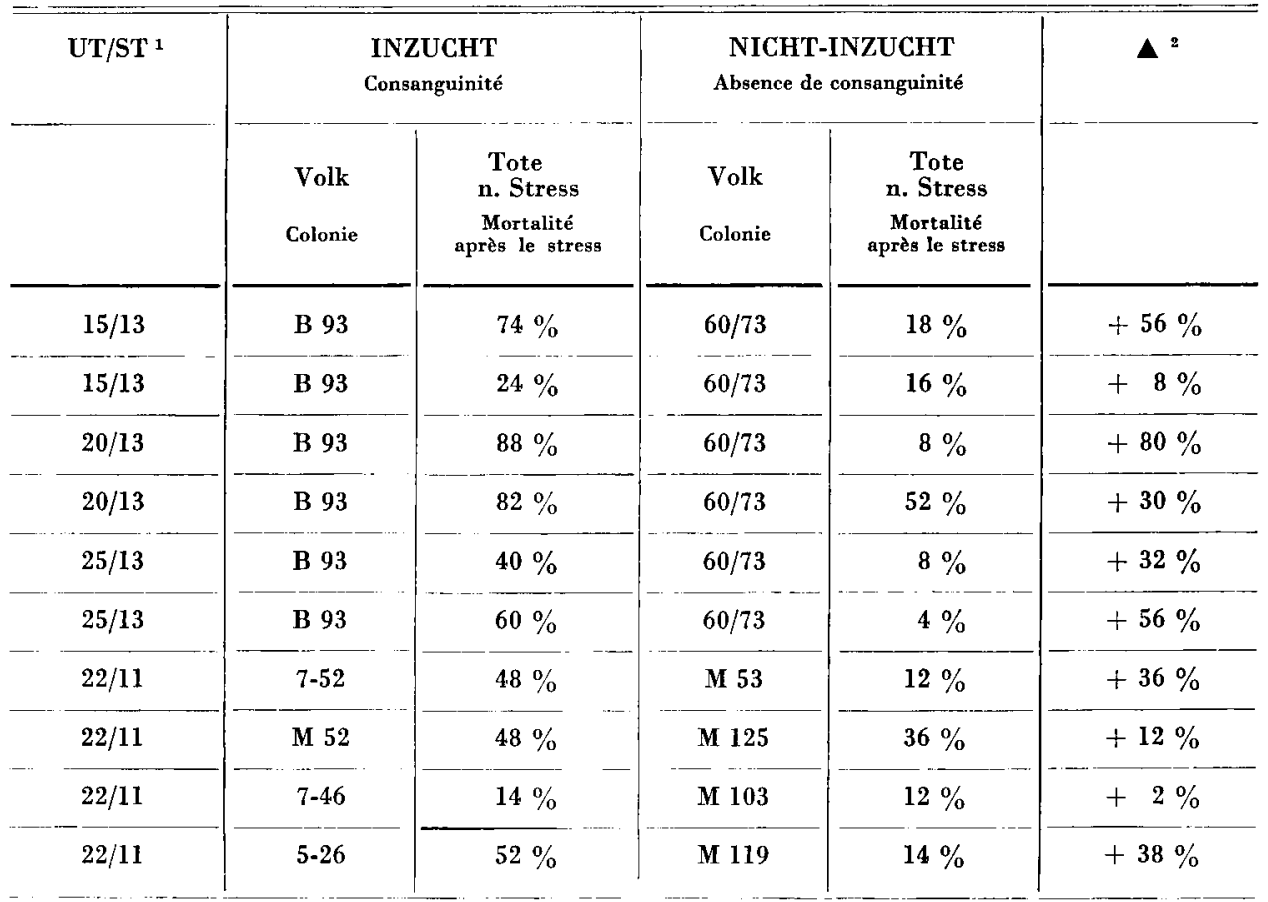

$1 \mathrm{UT} / \mathrm{ST}=$ Umgebungstemperatur während der Versuche/Stresstemperatur (in ${ }^{\circ} \mathrm{C}$ )

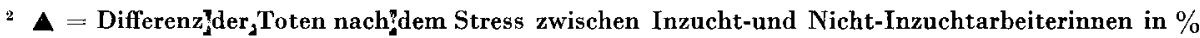

$1 \mathrm{UT} / \mathrm{ST}=$ température ambiante durant l'expérience / température de stress (en ${ }^{\circ} \mathrm{C}$ ).

$2 \boldsymbol{A}=$ différence de mortalité après le stress entre les ouvrières consanguines et les ouvrières non consanguines en $\%$. 
stress untersucht. Die Kästchen waren während der Stressversuche paarweise angeordnet; es wurden jeweils die Tiere der Kästchen verglichen, die in den Versuchen gleichen Bedingungen ausgesetzt waren. In Tabelle 1 sind die Verluste an Arbeiterinnen wiedergegeben. In allen Fällen überlebten weniger Inzucht- als Nicht-Inzuchtbienen.

\section{DISKUSSION}

Wie in der Einleitung erläutert, sind mehrere Mechanismen an der Temperaturregulierung im Bienenvolk beteiligt. Meine Versuchsanordnung war so gewählt, daß nur die durch Kälte hervorgerufenen Reaktionen getestet wurden, denn die Temperatur in den Völkern wurde im zeitigen Frühjahr bei niedrigen Außentemperaturen gemessen und die Temperaturstufen der Kästchenversuche lagen zwischen $15{ }^{\circ} \mathrm{C}$ und $25^{\circ} \mathrm{C}$. Bei den Kästchenversuchen steht der Mechanismus der Stoffwechselwärmeabgabe zur Temperaturregulierung im Vordergrund, da die Traubenbildung bei so kleinen Gruppen von Bienen nur eine untergeordnete Rolle spielt. Bei der Temperaturregulierung im Volk kommt sowohl die Traubenbildung als auch die Stoffwechselwärmeerzeugung zur Wirkung; über die Traubenbildung lassen sich jedoch auch im Volk nur indirekte Aussagen machen, da die Temperatur nur im Zentrum des Brutnestes gemessen wurde. Um die Traubenbildung direkt zu erfassen, hätte man mit zahlreichen Thermoelementen an allen Waben eines Volkes messen müssen; dies lag jedoch nicht im Bereich des Möglichen.

Die Ergebnisse der Messungen in den Völkern zeigen, daß die NichtInzuchtbienen den Inzuchtbienen in ihrer Fähigkeit, die Temperatur zu regulieren, überlegen sind. Sie erreichen im Mittel signifikant höhere Werte und können diese auch stabiler halten. Da die Traubenbildung bei der Stabilhaltung der Temperatur eine große Rolle spielt, deuten die Ergebnisse darauf hin, daß der Mechanismus der Traubenbildung in Nicht-Inzuchtvölkern effektiver funktioniert als in Inzuchtvölkern. Zudem konnte bei den Inzuchtvölkern eine Abhängigkeit von der Umgebungstemperatur nachgewiesen werden, während die Nicht-Inzuchtvölker keine Abhängigkeit von der Umgebungstemperatur zeigten. Dies wird auch durch die Ergebnisse der Kästchenversuche bei $15{ }^{\circ} \mathrm{C}, 20{ }^{\circ} \mathrm{C}$ und $25{ }^{\circ} \mathrm{C}$ bestätigt, in denen zumindest bei $20^{\circ} \mathrm{C}$ und $25{ }^{\circ} \mathrm{C}$ die Nicht-Inzuchtarbeiterinnen eine signifikant höhere Heizleistung erbringen als die Inzuchtarbeiterinnen. In den Kästchenversuchen wird die Stoffwechselwärmeerzeugung gemessen, also die vom Individuum abhängige Komponente der Temperaturregulierung. Die Ergebnisse zeigen, daß die Inzuchtbienen den Nicht-Inzuchtbienen in der Stoffwechselwärmeerzeugung unterlegen sind.

Die Unterschiede, die in der Fähigkeit der Temperaturregulierung zwischen Inzucht- und Nicht-Inzuchtbienen gefunden wurden, müssen durch die 
Unterschiede in der genetischen Variabilität der Tiere bedingt sein. Dies geht besonders deutlich aus der Tatsache hervor, daß eng verwandte ingezüchtete und nicht-ingezüchtere Völker signifikante Unterschiede in ihrer Fähigkeit zur Temperaturregulierung aufweisen. Es könnte nun argumentiert werden, daß bei den Inzuchtarbeiterinnen neben einem Mangel an Regulierfähigkeit eine Verschiebung des Sollwertes für die Temperatur, die aufgeheizt werden soll, vorliegt. Folgende Ergebnisse sprechen jedoch mehr für eine geringere Regulierfähigkeit als für einen verschobenen Sollwert bei den Inzuchtarbeiterinnen :

1. Bei den Messungen im Volk erreichten die Inzuchtbienen bei niedrigster Umgebungstemperatur auch die niedrigste Temperatur im Volk, während bei höherer Umgebungstemperatur auch höhere Temperaturen im Volk erreicht wurden. Die Nicht-Inzuchtvölker erreichten demgegenüber unabhängig von der Umgebungstemperatur immer etwa gleich hohe Temperaturen; sie konnten die erreichten Temperaturen auch stabiler halten als die Inzuchtvölker. Wäre der Sollwert bei der Inzucht verstellt, so würde man auch bei ihnen gleichbleibende Brutnesttemperaturen erwarten und es dürfte keine Abhängigkeit von der Umgebungstemperatur auftreten.

2. In den Streßversuchen, bei denen es für die Bienen darum ging, genügend Wärme zu erzeugen, um die schlechten Umweltbedingungen zu überstehen, starben immer mehr Inzucht- als Nicht-Inzuchtbienen. Die Streßtemperaturen lagen so niedrig, daß es für die Bienen nur um das Überleben gehen konnte, nicht aber darum, einen erwünschten Sollwert einzustellen. Die NichtInzuchtarbeiterinnen waren offensichtlich in der Lage, eine bessere Heizleistung zu erbringen als die Inzuchtarbeiterinnen.

3. Bei den Kästchenversuchen konnte beobachtet werden, daß die Inzuchttiere oft weniger aktiv waren als die Nicht-Inzuchttiere; wahrscheinlich bedeutet mehr Aktivität auch mehr Wärmeabgabe.

Diese Argumente sprechen dafür, daß die schlechtere Temperaturregulierung der Inzuchtbienen auf eine schlechtere Regulierfähigkeit zurückzuführen ist. Eine Verschiebung des Sollwerts bei der Inzucht erscheint demnach unwahrscheinlich, kann aber mit den vorliegenden Daten nicht ausgeschlossen werden. Um herauszufinden, ob der Sollwert der Inzuchtarbeiterinnen niedriger liegt, müßte man nicht nur Versuche bei niedriger sondern auch bei hoher Umgebungstemperatur machen. Bei verschobenem Sollwert müßten die Inzuchtarbeiterinnen die Brutnesttemperatur bei niedrigen und hohen Umgebungstemperaturen auf ein niedrigeres Niveau einregulieren als die NichtInzuchtarbeiterinnen.

Die schlechtere Regulierfähigkeit der Inzuchtarbeiterinnen muß auf einer geringeren Leistungsfähigkeit der Individuen bezüglich der Erzeugung von Stoffwechselwärme beruhen, sowie auf einer weniger effektiven Trauben- 
bildung des Kollektivs aller Bienen. Das Homozygotwerden vieler Loci im Genom bedeutet für das Volk also einen Verlust an Pufferungskapazität gegenüber störenden Umweltein flüssen.

Die Temperaturregulierung ist für die Bienenvölker zweifellos ein lebenswichtiger Faktor. Ohne Temperaturregulierung könnte kein Volk die kalten Winter unserer Breiten überleben, und würde die Brutnesttemperatur trotz der Schwankungen der Umgebungstemperatur nicht bei etwa $35^{\circ} \mathrm{C}$ gehalten, so wäre die Entwicklung der Larven sehr gefährdet. In der vorliegenden Arbeit konnte gezeigt werden, daß der Heterozygotiegrad der Arbeiterinnen einen erheblichen Einfluß auf das Merkmal der Temperaturregulierung besitzt. Es konnte damit ein quantitativ meßbares Merkmal gefunden werden, in dem die Inzuchtbienen den Nicht-Inzuchtbienen unterlegen sind. Bei früheren Untersuchungen zur Entwicklungshomöostasis konnte nachgewiesen werden, daß ingezüchtete Bienen auch in morphogenetischen Merkmalen eine weniger gute Regulierfähigkeit besitzen als nicht-ingezüchtete Bienen (BrücKner 1973b, 1975). Die Verringerung der genetischen Variabilität wirkt sich bei den Arbeiterinnen also auf die Loci aus, die für die physiologische Homöostasis und die Entwicklungshomöostasis der Individuen von Bedeutung sind. Es wäre jedoch denkbar, daß nicht alle Loci der Arbeiterinnen bei Homozygotwerden eine verminderte Merkmalsausprägung verursachen, da im haplodiploiden System der Honigbiene zumindest zeitweilig ein Teil der Population aus hemizygoten Drohnen besteht. Diese besitzen nur den halben Chromosomensatz und haben daher, verglichen mit den heterozygoten Arbeiterinnen, eine erheblich reduzierte genetische Variabilität; zwar müssen sie funktionsfähige Organismen sein, z. B. für den Flug zu den Drohnensammelplätzen und bei der Begattung der Königin, doch verhalten sie sich passiv bei den Arbeiten im Stock, so auch bei der Temperaturregulierung. Es ist bekannt, daß Drohnen ausserhalb des Volkes bei einer Umgebungstemperatur von etwa $20{ }^{\circ} \mathrm{C}$ bereits nach kurzer Zeit an Aktivität verlieren, während sie im Brutschrank bei $30{ }^{\circ} \mathrm{C}$ mehrere Stunden lang aktiv bleiben. Die Fähigkeit zur Temperaturregulierung ist also an die Heterozygotie der Individuen gebunden, es handelt sich jedoch um ein Merkmal, für das keine Selektion bei den Drohnen besteht. Wie verhält es sich aber mit Merkmalen, die bei Arbeiterinnen und Drohnen unter Selektionsdruck stehen, so z.B. die Augengröße oder die Körpergröße? Es wäre interessant festzustellen, ob sich die Ausbildung solcher Merkmale bei Inzuchtarbeiterinnen im Vergleich mit normal heterozygoten Arbeiterinnen in Richtung auf die der Drohnen verschiebt, ob z. B. die Augengröße der Arbeiterinnen mit hohem Homozygotiegrad zunimmt.

Was die Auswirkungen der Inzucht auf das Temperaturregulierungsvermögen der Arbeiterinnen betrifft, so wird deutlich, daß Inzucht von der Honig- 
biene vermieden werden muß, um für das Volk Jebenswichtige Fähigkeiten bei den Arbeiterinnen zu erhalten. Im Laufe der Evolution bildeten sich daher Verhaltensweisen aus, die Inzucht verhindern, indem die Fremdbesamung der Königin unter natürlichen Bedingungen sichergestellt wurde.

Eingegangen im Juni 1975.

Reçu pour publication en juin 1975.

\section{DANK}

Herrn Professor Dr. F. Ruttner möchte ich danken für die freundliche Überlassung des Bienenmaterials und die Ermöglichung eines mehrwöchigen Arbeitsaufenthaltes im Institut für Bienenkunde in Oberursel bei Frankfurt, sowie ihm und Professor Dr. J. Jacobs für die anregenden Diskussionen.

\section{RÉSUMÉ}

On a comparé chez des colonies consanguines et non consanguines la capacité à réguler la température. La faculté de thermorégulation est un paramètre de l'homéostasie des animaux.

Les ouvrières consanguines possédaient un fort degré d'homozygotie, qui avait été obtenu par insémination artificielle des reines; chez celles-là le coefficient de consanguinité $F$ s'élevait à $87,5 \%$, tandis que les ouvrières non consanguines étaient issues de reines normalement fécondées $(F=0 \%)$. On a effectué des mesures sur des colonies installées en plein air et sur des groupes de 50 ouvrières en chambre climatisée.

Lors des mesures sur les colonies on a placé au centre du nid à couvain les éléments thermiques d'un appareil de mesure électronique; en même temps que la température de chaque colonie non consanguine on relève aussi celle d'une ou deux colonies consanguines et la température ambiante. On a effectué les mesures au début du printemps sur une totalité de 7 colonies consanguines et de 3 non consanguines.

Pour les mesures sur les petits groupes d'abeilles, on a pris des ouvrières dans 9 colonies consanguines et 5 non consanguines. On les a groupées à raison de 50 originaires d'une même colonie dans des cagettes en plexiglas placées dans une étuve à couvain pour les faire éclore. On les a laissées là 10 jours pour qu'elles atteignent l'âge correspondant à la fonction de nourrice. Pendant cette période ainsi que durant toute l'expérience on fournit aux abeilles du pollen frais, du miel et de l'eau à volonté. Pendant les expériences les abeilles en chambre climatisée ont été conservées à $15{ }^{\circ} \mathrm{C}, 20^{\circ} \mathrm{C}, 22{ }^{\circ} \mathrm{C}$ et $25{ }^{\circ} \mathrm{C}$. Les mesures ont été effectuées à l'aide d'un appareil portatif qui possédait une antenne que l'on pouvait introduire dans les cagettes.

En liaison avec les mesures de thermorégulation on a testé la faculté de survie des petits groupes d'abeilles en état de stress thermique (basse température). Les mesures ont fait apparaître que, dans les colonies comme dans les petits groupes, les ouvrières non consanguines sont supérieures aux ouvrières consanguines quant à leur capacité à réguler la température. Les abeilles non consanguines atteignent des températures nettement plus élevées que les abeilles consanguines et sont mieux capables de les stabiliser à l'intérieur de la ruche. Les colonies non consanguines atteignent des températures dans le nid à couvain sensiblement aussi élevées, quelle que soit la température ambiante, tandis que les températures du nid à couvain des colonies consanguines sont d'autant plus basses que la température ambiante est plus basse.

Parmi les petits groupes d'abeilles conservés à $20^{\circ} \mathrm{C}, 22{ }^{\circ} \mathrm{C}$ et $25{ }^{\circ} \mathrm{C}$, les ouvrières non consanguines atteignent des températures nettement plus élevées que les ouvrières consan- 
guines. En état de stress thermique (basse température), les ouvrières non consanguines survivent toujours plus longtemps que les consanguines.

La thermorégulation moins bonne que réalisent les ouvrières consanguines est attribuée au fait que la réduction de la variabilité génétique perturbe l'équilibre interne (homéostasie physiologique) des animaux, de telle sorte que les mécanismes de la thermorégulation ne fonctionnent plus de la même façon que chez les animaux normalement hétérozygotes. Par exemple, les ouvrières consanguines produisent, dans des conditions semblables, moins de chaleur métabolique que les ouvrières non consanguines. On discute de savoir si à côté d'une mauvaise thermorégulation il peut y avoir également déplacement de la valeur théorique chez les abeilles consanguines. Pourtant les données indiquent plus une mauvaise capacité de régulation qu'une valeur théorique déplacée.

Une bonne thermorégulation est une question vitale pour les colonies d'abeilles; la consanguinité conditionne une dégradation de ce caractère; ceci doit être une raison pour que la consanguinité ne se produise pas chez les abeilles dans des conditions naturelles.

\section{LITERAT URVERZEICHNIS}

BrǘcKner D., 1973a. - Reduction of biochemical polymorphisms in honeybees (Apis mellifica). Experientia, 30, 618-619.

433-434.

1973b. - Homeostasis in honeybees (Apis m. carnica). Die Naturwiss., 60 (9),

1975. - The in fluence of genetic variability on wing symmetry in honeybees (Apis mellifica). Evolution (in press).

Drescher W., 1969. - in : Die instrumentelle Besamung der Bienenkönigin. Herausg. F. Ruttner, Apimondia, 58-61.

Free J. B., SPENCER-Booth Y., 1958. - Observations on the temperatureregulation and food consumption of honeybees (Apis mellifera). Exper. Biol., 35 (4) 930-937.

Free J. B., 1959. - Temperature regulation by honeybees. Bee World, 40 (7) 173-177.

Gates B. N., 1914. - The temperature of the bee colony. Bull. U.S. Dep. Agric., 96, 1-29.

Hrmmer A., 1932. - Die Temperaturverhältnisse bei den sozialen Hymenopteren. Biol. Rev., 7, 224-253.

Lerner I. M., 1954. - Genetic Homeostasis. New York, John Wiley.

Lewontiv R. C., 1956. - Studies on homeostasis and heterozygosity. I. General considerations. Abdominal bristle. Number in second chromosome homozygotes of Drosophila melanogaster. Am Nat., 90, 853, 237-255.

Lindauer M., 1954. - Temperaturregulierung und Wasserhaushalt im Bienenstaat. Z. vergl. Physiol., 36, 391-432.

MACKensen O., 1951. - Viability and sex-determination in the honeybee (Apis mellifera L.). Genetics 36, 5, 500-509.

WiLson E. O., 1971. — The Insect Societies. Belknap Press, Cambridge Mass., chapter 16.

Woyke J., 1965. - Genetic proof of the origin of drones from fertilized eggs of the honeybee J. Apic. Res., 4, (1) 7-11. 